\title{
The Effects of New Alibernet Red Wine Extract on Nitric Oxide and Reactive Oxygen Species Production in Spontaneously Hypertensive Rats
}

\author{
Alexey Kondrashov, ${ }^{1}$ Stanislava Vranková, ${ }^{2}$ Ima Dovinová, ${ }^{2}$ Rudolf Ševčík, ${ }^{3}$ \\ Jana Parohová, ${ }^{2}$ Andrej Barta, ${ }^{2}$ Olga Pecháňová, ${ }^{2}$ and Maria Kovacsová ${ }^{2}$ \\ ${ }^{1}$ Department of Medical Biochemistry, First Faculty of Medicine, Charles University in Prague, 12108 Prague, Czech Republic \\ ${ }^{2}$ Centre of Excellence for Regulatory Role of Nitric Oxide in Civilization Diseases, Institute of Normal and Pathological Physiology, \\ Slovak Academy of Sciences, 81371 Bratislava, Slovakia \\ ${ }^{3}$ Department of Food Preservation, Faculty of Food and Biochemical Technology, Institute of Chemical Technology, \\ 16628 Prague, Czech Republic
}

Correspondence should be addressed to Alexey Kondrashov, alexey.kondrasov@lf1.cuni.cz

Received 9 March 2012; Accepted 16 April 2012

Academic Editor: Luciano Pirola

Copyright ( 2012 Alexey Kondrashov et al. This is an open access article distributed under the Creative Commons Attribution License, which permits unrestricted use, distribution, and reproduction in any medium, provided the original work is properly cited.

We aimed to perform a chemical analysis of both Alibernet red wine and an alcohol-free Alibernet red wine extract (AWE) and to investigate the effects of AWE on nitric oxide and reactive oxygen species production as well as blood pressure development in normotensive Wistar Kyoto (WKY) and spontaneously hypertensive rats (SHRs). Total antioxidant capacity together with total phenolic and selected mineral content was measured in wine and AWE. Young 6-week-old male WKY and SHR were treated with AWE (24,2 mg/kg/day) for 3 weeks. Total NOS and SOD activities, eNOS and SOD1 protein expressions, and superoxide production were determined in the tissues. Both antioxidant capacity and phenolic content were significantly higher in AWE compared to wine. The AWE increased NOS activity in the left ventricle, aorta, and kidney of SHR, while it did not change NOS activity in WKY rats. Similarly, increased SOD activity in the plasma and left ventricle was observed in SHR only. There were no changes in eNOS and SOD1 expressions. In conclusion, phenolics and minerals included in AWE may contribute directly to increased NOS and SOD activities of SHR. Nevertheless, 3 weeks of AWE treatment failed to affect blood pressure of SHR.

\section{Introduction}

Recent findings have supported the idea that natural bioactive compounds could be beneficial for the prevention and treatment of cardiovascular disease, although the benefits for individuals may depend on their genetic profile [1-7]. Among foodstuffs widely distributed in the human diet, red wine has attracted considerable interest as a potential source of bioactive compounds such as polyphenols and minerals [8-10].

To date several studies have proposed the mechanisms by which red wine could exert its beneficial effects toward cardiovascular disorders and hypertension in particular. These mechanisms mainly involve increased vasorelaxation and blood pressure reduction by enhancing nitric oxide synthase (NOS) activity and nitric oxide production [11-15]. Moreover, red wine could modulate activities of endogenous antioxidant enzymes, thereby enhancing the functions of body defense against the oxidative stress [16, 17].

Polyphenols and minerals are among the principal bioactive compounds present in red wines. While red wine polyphenolic compounds are well known for their direct free radical scavenging, antioxidant, anti-inflammatory, antiplatelet aggregation, and lipid metabolism improving activities $[18,19]$, the effects of minerals present in red wines have been studied much less. Red wine contains a number of trace elements such as magnesium, zinc, potassium, and manganese essential for the proper function of endogenous 
antioxidant system $[8,20]$. Mounting evidence suggests that minerals are able to counteract the development of cardiovascular, metabolic and other diseases $[21,22]$. The positive effects of minerals in humans are related to their ability to enhance the activity of the body's antioxidant defense system by catalyzing antioxidant enzymes. For instance, several minerals found in foodstuffs, such as copper, zinc, and manganese, are essential for activity of superoxide dismutase (SOD), a key antioxidant enzyme [23, 24].

The modulation of nitric oxide synthase activity seems to be the most promising tool by which different polyphenolic compounds could regulate the blood pressure. It has been documented that oral administration of red wine polyphenolic compound can reduce myocardial fibrosis, prevent aortic thickening, attenuate the increase of aortic reactivity to norepinephrine, prevent the decrease in acetylcholineinduced endothelium-dependent relaxation, and, in rats, reduce blood pressure during NO deficiency. These alterations were associated with increased NOS activity, a moderate increase in endothelial NOS expression, and reduction of oxidative stress, the factors which may be responsible for the beneficial effect of the red wine polyphenolic compounds $[25,26]$. Moreover, the study of Bernátová et al. demonstrated that red wine polyphenols (Provinols) even accelerate the decrease in blood pressure and improved structural and functional cardiovascular changes produced in developed form of $\mathrm{N}^{\mathrm{G}}$-nitro-L-arginine-methyl-ester (LNAME)-induced hypertension [27].

Despite considerable attention paid toward cardiovascular disorders, experimental studies are still lacking. This study provides comprehensive chemical analysis of both red wine and red wine extract in order to evaluate the effect of red wine compounds on NOS and SOD activities, eNOS and SOD1 expressions, and blood pressure development in normotensive and spontaneously hypertensive rats.

\section{Materials and Methods}

2.1. Chemicals. Gallic acid, Folin-Ciocalteau reagent, potassium peroxodisulfate, potassium superoxide, Trolox, and SOD assay kit were obtained from Sigma-Aldrich. The ABTS assay used a commercial kit Randox TAS was purchased from Randox Laboratories, Antrim, United Kingdom. Lucigenin purchased from Fluka. All the other reagents were of analytical grade.

The wine examined was obtained from the Slovak State Institute of Viniculture (Modra, Slovakia). Samples of Alibernet red wine were subjected to the process of dealcoholization, and concentration, producing an alcohol-free ten times concentrated Alibernet red wine extract (AWE).

\subsection{Alibernet Red Wine and AWE Characterization}

2.2.1. Measurement of Total Antioxidant Capacity. In the Alibernet wine and AWE samples, TAC was measured using spectrophotometric assays on a Thermo Spectronic Genesys 20 UV-visible spectrophotometer (Thermo Fisher Scientific, Waltham, MA, USA). TAC was determined using a commercial kit Randox TAS (radical ABTS; Randox Laboratories, Antrim, United Kingdom) according to the manufacture instructions. Amounts of antioxidant power are expressed in Trolox (6-hydroxy-2,5,7,8-tetramethylchroman-2-carboxylic acid) equivalents ( $\mathrm{mmol} / \mathrm{L})$.

2.2.2. Measurement of Total Phenolic Content. Total phenolic content was determined using Folin-Ciocalteau reaction [28] by spectrophotometric assay on a Thermo Spectronic Genesys 20 UV-visible spectrophotometer (Thermo Fisher Scientific, Waltham, MA, USA). Absorbance was measured at $760 \mathrm{~nm}$ using gallic acid as the standard. TPC is expressed in gallic acid equivalents (GAEs).

2.2.3. Determination of Minerals. The red wine and AWE elemental composition was determined by AAS method using acetylene/argon flame in Atomic Absorption Spectrometer (Varian Spectra AA 220 FS, Australia) for copper, zinc, selenium, and lead, ISE (Ion Selective Electrode) for potassium determination, and Photometry (Roche equipment, Modular E 170, Switzerland) for calcium, magnesium, phosphorus, and iron determination.

\subsection{Animal Studies}

2.3.1. Animals and Treatment. All procedures and experimental protocols were approved by the Ethical Committee of the Institute of Normal and Pathological Physiology, Slovak Academy of Sciences, and conform to the European Convention on Animal Protection and Guidelines on Research Animal Use.

Male 6-week-old normotensive Wistar Kyoto (WKY) and spontaneously hypertensive rats (SHR) were divided into the control groups and groups treated with AWE $(24,2 \mathrm{mg} / \mathrm{kg} / \mathrm{day})$ for 3 weeks $(n=6$ in each group). The extract was given in tap water. To ensure that each animal received the complete amount of AWE, the calculated amount of liquid extract was given to each cage in the appropriate volume of water and adjusted to the animal water consumption. Daily water consumption was estimated individually for every animal 1 week before the experiment. During the experiment, water consumption was controlled. All animals were housed at a temperature of $22-24^{\circ} \mathrm{C}$ and fed with a regular pellet diet ad libitum. Systolic blood pressure (SBP) was measured by a noninvasive method of tail-cuffplethysmography every week.

After 3 weeks of treatment, the animals were sacrificed and the body weight (BW) and heart weight (HW) were determined. Samples of the left ventricle, aorta, and kidney were used to determine NOS and SOD activities and eNOS and SOD1 protein expressions. Superoxide levels were measured in the left ventricle and aorta. Total antioxidant capacity and SOD activity were measured also in the plasma.

2.3.2. Total NOS Activity and eNOS Protein Expression. Total NOS activity was determined in crude homogenates of the $\mathrm{LV}$, aorta, and kidney by measuring the formation of $\left[{ }^{3} \mathrm{H}\right]-$ L-citrulline from $\left[{ }^{3} \mathrm{H}\right]$-L-arginine as previously described by 
Bredt and Snyder, with minor modifications by Pecháňová et al. $[29,30] .\left[{ }^{3} \mathrm{H}\right]-\mathrm{L}$-citrulline was measured with the Quanta Smart TriCarb Liquid Scintillation Analyzer (Packard Instrument company, Meriden, CT). Total NOS activity is expressed as pkat/g of proteins.

For eNOS protein expression, samples were prepared according to Pecháňová et al. [31]. Following the electrophoresis, proteins were transferred to nitrocellulose membranes and were probed with a polyclonal rabbit anti-eNOS antibody (Santa Cruz Biotechnology, Inc., USA). Bound antibody was detected using a secondary peroxidase-conjugated anti-rabbit antibody (Santa Cruz Biotechnology, Inc., USA). The bands were visualized using the enhanced chemiluminescence system (ECL, Amersham, UK) and analyzed densitometrically using Photo-Capt V.99 software.

2.3.3. Total SOD Activity and SOD1 Protein Expression. The SOD activity was analyzed using the SOD assay kit (Fluka). The absorbance was read at $450 \mathrm{~nm}$ using a microplate reader (Thermo Scientific Multiscan FC, Finland). SOD activity was calculated using activity of SOD standards, and results were expressed in $\mathrm{U} / \mathrm{mL}$ in plasma or $\mathrm{U} / \mathrm{mg}$ of protein in tissues.

For SOD1 protein expression, following the electrophoresis, proteins were transferred to nitrocellulose membranes and were probed with a polyclonal rabbit antiSOD1 antibody (Santa Cruz Biotechnology, Inc., USA). Bound antibody was detected using a secondary peroxidaseconjugated anti-rabbit antibody (Santa Cruz Biotechnology, Inc., USA). The bands were visualized using the enhanced chemiluminescence system (ECL, Amersham, UK) and analyzed densitometrically using Photo-Capt V.99 software.

2.3.4. Total Antioxidant Capacity of Plasma. TAC in plasma was determined using decolorisation assay and expressed as trolox equivalent antioxidant capacity (TEAC). Antioxidant capacity of samples affect changes in decolorisation of ABTS radical $\left(3 \mathrm{~min}, 37^{\circ} \mathrm{C}\right)$, measured spectrophotometrically at $720 \mathrm{~nm}$ [32]. Trolox (6-hydroxy-2,5,7,8tetramethylchroman-2-carboxylic acid) was used as standard and expressed in $\mathrm{mmol} / \mathrm{L}$.

2.3.5. Measurement of Superoxide Level. The superoxide $\left(\mathrm{O}_{2}{ }^{-}\right)$was evaluated using lucigenin-enhanced chemiluminscence [33]. The aorta and left ventricle were cut into small pieces of up to $15 \mathrm{mg}$ wet weight. After equilibration and adaptation to dark conditions, samples were added to lucigenin solution and measured every $30 \mathrm{~s}$ for $5 \mathrm{~min}$ in a Turner Designs TD-20/20 luminometer. Results are provided in relative luminescence unit/mg tissue (RLU/mg tissue).

2.4. Statistics. For Alibernet red wine and AWE characterization, data are presented as mean values \pm standard deviation (SD; $n=3)$. The statistical significance was evaluated by Pearson's test, which is suitable for small numbers of samples, using GraphPad Prism version 4 software (GraphPad Software, San Diego, CA, USA).

For animal studies, one-way analysis of variance and the Duncan test were used for statistical analysis. Values were
TABLE 1: Total antioxidant capacity and total phenolic content of Alibernet red wine and Alibernet wine extract.

\begin{tabular}{lcc}
\hline Sample & TAC mmol/L & Total phenols GAE mg/L \\
\hline Alibernet red wine & 35,82 & $2039 \pm 0,55$ \\
AWE & 376,38 & $24172 \pm 1,26$ \\
\hline
\end{tabular}

GAE: gallic acid equivalents, AWE: alibernet red wine extract.

considered to differ significantly if the probability value was less than $0.05(P<0.05)$. Results are given as mean \pm SD.

\section{Results}

\subsection{Alibernet Red Wine and AWE Characteristics}

3.1.1. Total Antioxidant Capacity and Phenolic Content. Antioxidant capacity of Alibernet red wine was slightly above $35 \mathrm{mmol} / \mathrm{L}$, whereas, in Alibernet wine extract, it reached $376 \mathrm{mmol} / \mathrm{L}$. This means that approximately 10 times higher TAC was determined in AWE in comparison to Alibernet red wine. The results are visualized in Table 1 . This also proves that the process of extraction had not affected the antioxidant properties of the red wine extract.

The total phenolic content of the Alibernet wine samples determined by the Folin-Ciocalteau colorimetric method was $2039 \mathrm{mg} / \mathrm{L}$ in Alibernet wine and above $24172 \mathrm{mg} / \mathrm{L}$ of gallic acid equivalents in Alibernet red wine extract.

Our research here establishes that dealcoholised Alibernet red wine extract has more than 11-fold higher total antioxidant capacity and total phenolic content compared to Alibernet red wine. This confirms that AWE has preserved all the essential properties of wine. This also reveals a significant positive relationship between total antioxidant capacity and the total phenolic content.

3.1.2. Mineral Content. The most predominant elements detected in Alibernet red wine and AWE were potassium $(\mathrm{K})$, zinc $(\mathrm{Zn})$, magnesium $(\mathrm{Mg})$, and calcium $(\mathrm{Ca})$. As with TAC and TPC, wine extract contains up to 10 -fold higher values of these minerals. The results are visualized in Table 2.

\subsection{Animal Studies}

3.2.1. Animal Characteristics. SHR had significantly lower body weight and higher blood pressure compared to normotensive WKY. Alibernet wine extract treatment failed to change both body weight and blood pressure of WKY and SHR. Heart weight did not differ between groups. Results are shown in Table 3.

\subsubsection{Total NOS Activity and eNOS Protein Expression.} The Alibernet wine extract did not change total NOS activities in the tissues of WKY rats. However, it was able to increase significantly NOS activity in the left ventricle (Figure 1(a)), aorta (Figure 2(a)), and kidney (Figure 3(a)) of SHR compared to untreated control rats. Endothelial NOS protein expression was not changed significantly after the 
TABLE 2: Elemental profile of Alibernet red wine and Alibernet wine extract (AWE).

\begin{tabular}{lccccccccc}
\hline Sample & $\mathrm{K} \mathrm{mg} / \mathrm{L}$ & $\mathrm{Mg} \mathrm{mg} / \mathrm{L}$ & $\mathrm{P} \mathrm{mg} / \mathrm{L}$ & $\mathrm{Ca} \mathrm{mg/L}$ & $\mathrm{Fe} \mathrm{mg/L}$ & $\mathrm{Cu} \mathrm{mg} / \mathrm{L}$ & $\mathrm{Se} \mu \mathrm{g} / \mathrm{L}$ & $\mathrm{Pb} \mu \mathrm{g} / \mathrm{L}$ & $\mathrm{Zn} \mathrm{mg/L}$ \\
\hline Alibernet red wine & 1387,01 & 131,14 & 108,97 & 69,54 & 1,54 & $\mathrm{ND}$ & $\mathrm{ND}$ & $\mathrm{ND}$ & 354,30 \\
AWE & 9069,51 & 1334,07 & $\mathrm{ND}$ & 657,31 & 24,60 & 0,26 & $\mathrm{ND}$ & 7,83 & 5463,92 \\
\hline
\end{tabular}

Data are expressed as mean values $(n=3)$. ND: not detected.

TABLE 3: Animal characteristics: body weight, heart weight, and blood pressure of WKY and SHR.

\begin{tabular}{lcccc}
\hline & WKY-C $(n=6)$ & WKY + AWE $(n=6)$ & SHR-C $(n=6)$ & SHR+AWE $(n=6)$ \\
\hline Body weight $(\mathrm{g})$ & $249 \pm 13$ & $253 \pm 11$ & $211 \pm 12^{*}$ & $216 \pm 11^{*}$ \\
Heart weight $(\mathrm{g})$ & $0,809 \pm 0,044$ & $0,841 \pm 0,070$ & $0,791 \pm 0,046$ & $0,812 \pm 0,050$ \\
Blood pressure (mmHg) & $107 \pm 8$ & $108 \pm 6$ & $167 \pm 12^{*}$ & $176 \pm 14^{*}$ \\
\hline
\end{tabular}

WKY-C: control Wistar Kyoto rats; WKY + AWE: Wistar Kyoto rats treated with the Alibernet wine extract; SHR-CL: control spontaneously hypertensive rats; SHR + AWE: spontaneously hypertensive rats treated with the Alibernet wine extract. ${ }^{*} P<0.05$ SHR versus WKY.

AWE treatment in both WKY and SHR (Figures 1(b), 2(b), and $3(\mathrm{~b}))$.

3.2.3. SOD Activity and SOD1 Protein Expression. The Alibernet wine extract treatment failed to affect SOD activities as well as SOD1 protein expressions in the tissues of WKY rats. On the other hand, AWE treatment significantly increased SOD activity in the LV of SHR (by 54\%; Figure 4(a)). However, it had no effect on left ventricular SOD1 protein expression (Figure 4(b)). Similarly, AWE treatment failed to affect SOD activity and SOD1 protein expression in the aorta, kidney, and plasma of SHR (data not shown).

3.2.4. Total Antioxidant Capacity of Plasma. Consistent with unchanged plasma SOD activity, there were no changes in the total antioxidant capacity of plasma in both WKY and SHR groups after AWE treatment (data not shown).

3.2.5. Measurement of Superoxide Level. Despite an increased tendency of superoxide level in the left ventricle and aorta of SHR, there were no significant changes between the strains. Similarly, AWE treatment did not reveal any significant changes in both LV and aorta of Wistar Kyoto and spontaneously hypertensive rats (Table 4).

\section{Discussion}

Emerging evidence suggests that minerals as well as polyphenolic compounds could play an important role in the proper functions of the endogenous antioxidant system as well as nitric oxide synthase activity.

Here, for the first time, an approach of simultaneous analysis of total phenolic content, minerals, and antioxidative capacities was employed to provide complex assessment of the red wine extract used in the experimental animal model. It has been confirmed that the Alibernet wine extract has up to ten times higher amounts of polyphenols and minerals compared to the Alibernet red wine. These results allowed us to conclude that processing the wine to obtain the extract did not have the negative impact on the content of minerals and phenolics of interest to present study. Moreover, we confirmed a strong positive relationship between the total antioxidant capacity and the content of total phenolic and minerals in both wine and the wine extract. These results are consistent with the findings published for red wines by other investigators [34, 35]. Those authors have experimentally verified correlation between polyphenolic content and the total antioxidant activity. In addition to analysis of polyphenolic content, we have also studied mineral content, which to a certain extent could affect the total antioxidant capacity in the red wine extract as it was shown in Table 1.

Minerals contained in foodstuffs play an important role in the body homeostasis. A growing amount of clinical evidence indicates relationships between levels of minerals and progression of certain diseases. Many recent studies documented the ability of selected minerals to counteract the development of cardiovascular, metabolic, and other diseases [36-40]. Positive effects of minerals in humans are related to their ability to enhance the activity of antioxidant defense system by catalyzing antioxidant enzymes. Several minerals found in foodstuffs, such as copper, zinc, and manganese, are essential for proper activity of superoxide dismutase, a key antioxidant enzyme [41, 42]. Experimental studies with zinc supplementation have revealed an augmentation of catalase, glutathione-s-transferase, and SOD activity after zinc supplementation to rats for 4 months [43].

Since results of chemical analysis of Alibernet wine extract showed relatively high concentrations of minerals, this may possibly contribute to changes in the activity of different antioxidant enzymes and/or nitric oxide synthase. Thus, SOD activity and SOD1 protein expression, NOS activity, and eNOS protein expression were analyzed together in experimental animals after Alibernet wine extract treatment. Western Blot analysis did not shown any significant changes in SOD1 and eNOS protein expressions after AWE treatment in the tissues investigated. On the other hand, AWE treatment increased significantly SOD activity in left ventricle and NOS activity in the LV, aorta, and kidney in the spontaneously hypertensive rats.

Three forms of SOD are present in humans and in all other mammals: cytoplasmic SOD1, mitochondrial SOD2, and extracellular SOD3 [44]. SOD1 is a Cu-Zn-dependent 
TABLE 4: Level of superoxide in the aorta and left ventricle after treatment with AWE.

\begin{tabular}{lcccr}
\hline & WKY-C & WKY + AWE & SHR-C & SHR + AWE \\
\hline Superoxide level in aorta RLU/mg & $3,52 \pm 1,25$ & $5,66 \pm 1,55$ & $6,36 \pm 2,47$ & $7,11 \pm 1,82$ \\
Superoxide level in LV RLU/mg & $6,65 \pm 1,60$ & $8,12 \pm 1,93$ & $7,68 \pm 1,67$ & $8,01 \pm 2,81$ \\
\hline
\end{tabular}

WKY-C: control group; WKY + AWE: animals treated with the Alibernet wine extract;

SHR-C: control group; SHR + AWE: animals treated with the Alibernet wine extract.

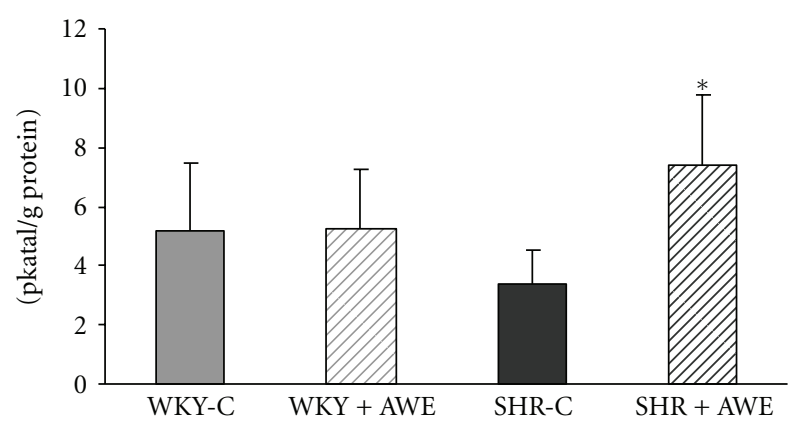

(a)

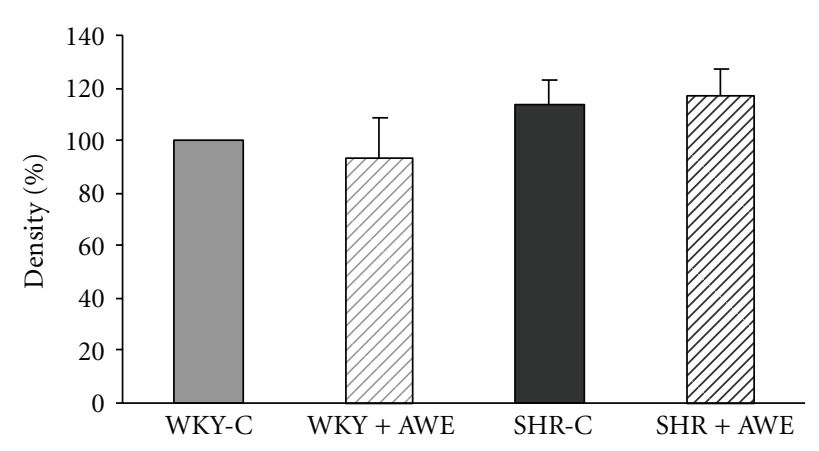

(b)

FIgURE 1: The effect of Alibernet red wine extract (AWE) treatment on total nitric oxid synthase (NOS) activity (a) and eNOS protein expression (b) in the left ventricle (LV) of Wistar Kyoto rats (WKY) and spontaneously hypertensive rats (SHR). $P<0.05$ as compared to respective control. WKY-C: control group; WKY + AWE: animals treated with the Alibernet wine extract; SHR-C: control group; SHR + AWE: animals treated with the Alibernet wine extract.

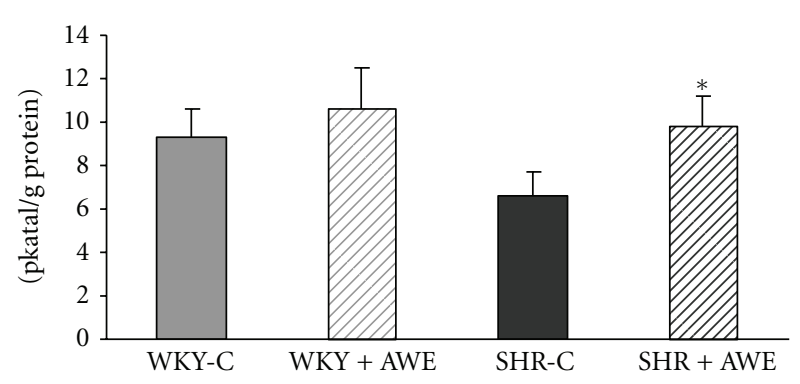

(a)

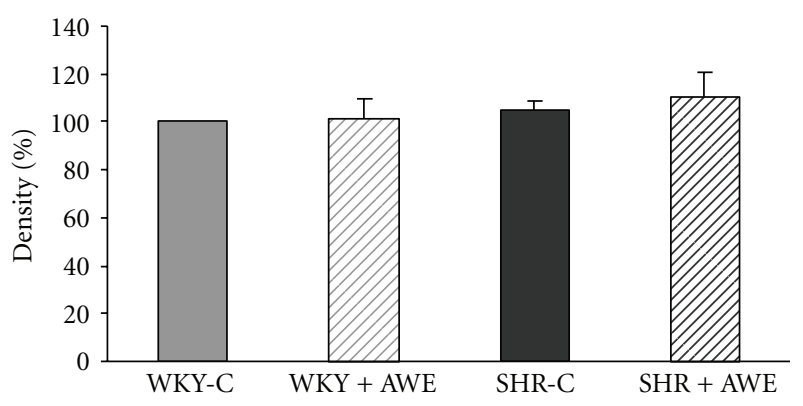

(b)

FIgURE 2: The effect of Alibernet red wine extract (AWE) treatment on total nitric oxid synthase (NOS) activity (a) and eNOS protein expression (b) in the aorta of Wistar Kyoto rats (WKY) and spontaneously hypertensive rats (SHR). $P<0.05$ as compared to respective control. WKY-C: control group; WKY + AWE: animals treated with the Alibernet wine extract; SHR-C: control group; SHR+AWE: animals treated with the Alibernet wine extract.

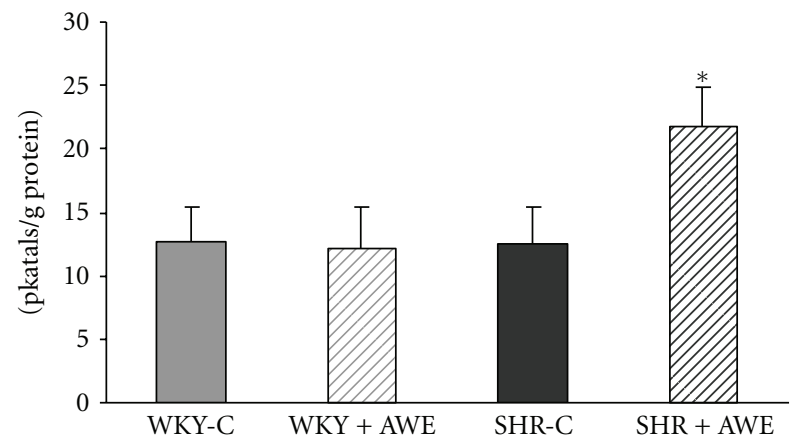

(a)

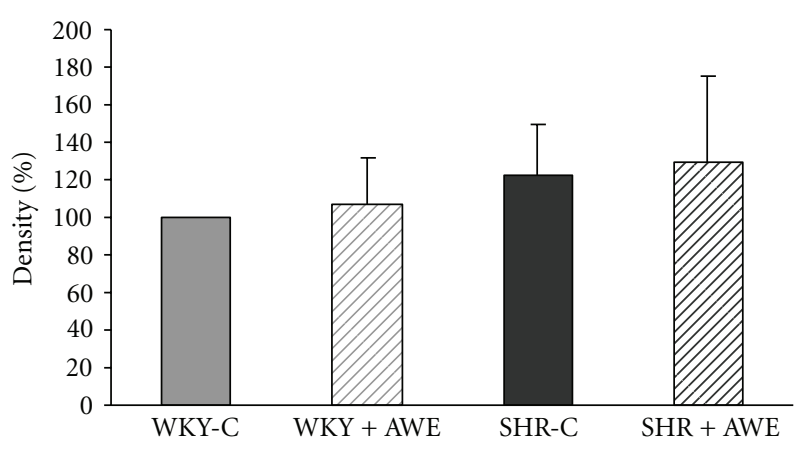

(b)

FIGURE 3: The effect of Alibernet red wine extract (AWE) treatment on total nitric oxid synthase (NOS) activity (a) and eNOS protein expression (b) in the kidney of Wistar Kyoto rats (WKY) and spontaneously hypertensive rats (SHR). $P<0.05$ as compared to respective control. WKY-C: control group; WKY + AWE: animals treated with the Alibernet wine extract; SHR-C: control group; SHR + AWE: animals treated with the Alibernet wine extract. 


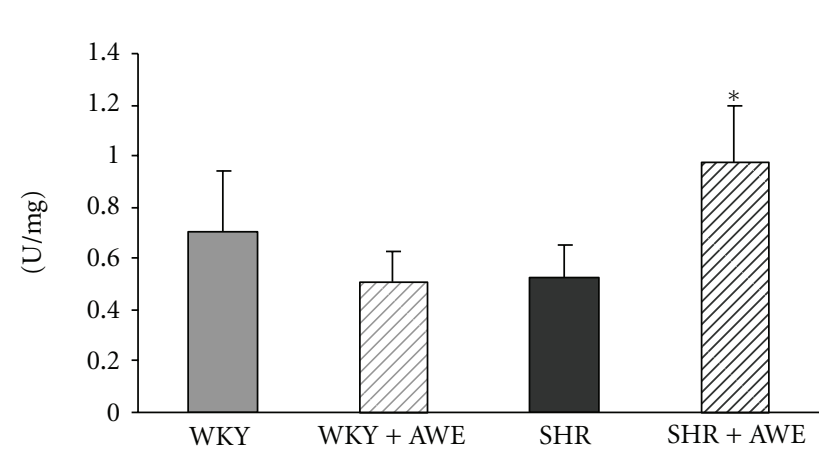

(a)

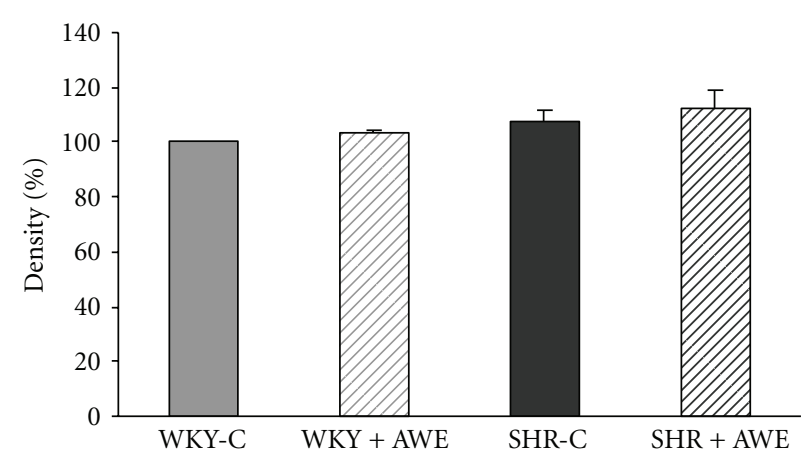

(b)

FIgURE 4: The effect of Alibernet wine extract (AWE) on superoxide dismutase (SOD) activity (a) and SOD1 protein expression (b) in the left ventricle (LV) of Wistar Kyoto rats (WKY) and spontaneously hypertensive rats (SHR). $P<0.05$ as compared to untreated animals. WKY-C: control group; WKY + AWE: animals treated with the Alibernet wine extract; SHR-C: control group; SHR + AWE: animals treated with the Alibernet wine extract.

isoform and is highly upregulated in the endothelial cells [45]. For this reason, SOD1 protein expression was analyzed. Since AWE treatment did not affect the level of SOD1 protein expression, we suppose that elevated level of $\mathrm{Zn}$ in AWE may directly increase SOD activity. We assume there is no increase in expression of SOD2 or SOD3 proteins after wine extract treatment. Of course, the polyphenol compounds involved in the extract may also contribute to increased SOD activity. These results are consistent with the recent study by Fernández-Pachón et al. [17] where an increased activity of SOD in human erythrocytes was observed after seven days of red wine consumption.

Zinc is one of the most important and ubiquitous trace elements in the body with significant antioxidant activity. Zinc is an important mineral in preventing free radical formation, protecting biological structures from damage and boosting the immune response [42, 46]. Moreover, Zhou et al. [47] have proven the key role of zinc in the proper function of nitric oxide synthase. In fact, NOS is catalytically active only as a dimer consisting of two subunits, the association of which is stabilized by tetrahedral coordination of $\mathrm{Zn}$ by thiol ligands at the dimer interface. That is why we also suggest that increased NOS activity after AWE treatment may be attributed to zinc. Besides zinc, AWE treatment could also increase the level of calcium-another important element necessary for endothelial NOS activation [48]. Our suggestion is confirmed by the fact that Western Blot analysis did not reveal any significant changes in endothelial NOS protein expression after AWE treatment of SHR. Thus $\mathrm{Zn}, \mathrm{Ca}$, and polyphenol compounds of AWE most probably directly increased NOS activity. We assume there is no increase in expression of the neuronal or inducible NOS proteins in the tissues investigated. LópezSepúlveda et al. [49] also did not see increased eNOS expression in the aorta of SHR after red wine polyphenol treatment. However, several other papers using different models documented increased eNOS expression after red wine polyphenol administration $[15,25]$. We assume that differences in polyphenolic consumption, different models as well as different treatment duration may be responsible for differences in eNOS protein expression.
Our results demonstrating increase in NOS activity in all samples investigated are consistent with the recent publications by Auger et al. [13] and Madeira et al. [15] where the authors found elevated NOS activity after using different red wine extracts. A recent study of Khoo et al. [50] focused on vascular responses to the polyphenolic compoundquercetin using a combination of biochemical and vasoactive criteria has also confirmed a positive correlation between quercetin concentration and increased NO bioavailability in endothelial cells. This study has confirmed the ability of polyphenolic compounds to improve endothelial cell function by stimulation of endothelial NOS in nitric oxide production.

Alibernet red wine extract increased both NOS and SOD activities only in rats with spontaneous hypertension, where the abovementioned harmful effects are present. This means that polyphenols and minerals may affect only pathological mechanisms while they have no effect under normal conditions. Similar effects of red wine polyphenols were described in L-NAME-induced hypertension [25]. Nevertheless, in our study, increased SOD and NOS activities after 3 weeks of AWE treatment failed to affect blood pressure of SHR.

The authors of several papers on this topic showed reduction of blood pressure after administration of red wine polyphenols to SHR. However, duration of the treatment was generally longer than three weeks. López-Sepúlveda et al. [49] documented reduction of hypertension and vascular dysfunction in SHR after red wine polyphenols treatment ( $40 \mathrm{mg} / \mathrm{kg}$ by gavage) for 5 weeks. Chan et al. [51] also found improved endothelium-dependent dilation after 10 -week oral administration of red wine polyphenols $(100 \mathrm{mg} / \mathrm{kg} / \mathrm{day})$ to SHR. On the other hand, Botden et al. [52] in a study on humans did not see decreased peripheral or central blood pressure in subjects with high-normal blood pressure or grade 1 hypertension after red wine polyphenol intake at two different dosages for 4 weeks. Concerning our study, increased SOD and NOS activities after 3 weeks of AWE treatment failed to affect blood pressure of SHR. We assume that prolongation of AWE treatment could lead to the reduction of blood pressure as well. This, however, requires further investigation. 
In conclusion, our study documented that phenolics and minerals presented in Alibernet wine extract, and zinc especially, may contribute directly to increased NOS and SOD activities in the tissues of spontaneously hypertensive rats. This finding enables us to suggest that the positive effects of fruits and vegetables are related to the increase in activity of the endogenous antioxidant enzymes and enhancement of antioxidant effects. This occurs together with an increase in NO production, important for vasodilation and blood flow improvement. This is particularly important in the case of hypertension, where elevated free radical and cytokine production, chronic inflammation, and the suppression of NO production are present.

\section{Abbreviations}

AWE: Alibernet red wine extract

NOS: Nitric oxide synthase

SOD: Superoxide dismutase

WKY: Wistar Kyoto

SHR: Spontaneously hypertensive rats

TAC: Total antioxidant capacity

GAE: Gallic acid equivalents

TPC: Total phenolic content

TEAC: Trolox equivalent antioxidant capacity.

\section{Acknowledgment}

This study was elaborated within the project "ITMS 26240120006-Establishment of the Centre for the Research on Composite Materials for Structural, Engineering and Medical Applications-CEKOMAT II" supported from the R\&D program of ERDF and by Grant APVV-0742-10.

\section{References}

[1] L. Badimon, G. Vilahur, and T. Padro, "Nutraceuticals and atherosclerosis: human trials," Cardiovascular Therapeutics, vol. 28, no. 4, pp. 202-215, 2010.

[2] P. M. Kris-Etherton, K. D. Hecker, A. Bonanome et al., "Bioactive compounds in foods: their role in the prevention of cardiovascular disease and cancer," American Journal of Medicine, vol. 113, no. 9, supplement, pp. 71S-88S, 2002.

[3] H. E. Seifried, D. E. Anderson, E. I. Fisher, and J. A. Milner, "A review of the interaction among dietary antioxidants and reactive oxygen species," Journal of Nutritional Biochemistry, vol. 18, no. 9, pp. 567-579, 2007.

[4] I. Arts and P. Hollman, "Polyphenols and Disease Risk in Epidemiologic Studies," American Journal of Clinical Nutrition , vol. 81, supplement, pp. 317S-325S, 2005.

[5] V. B. Schini-Kerth, N. Étienne-Selloum, T. Chataigneau, and C. Auger, "Vascular protection by natural product-derived polyphenols: in vitro and in vivo evidence," Planta Medica, vol. 77, no. 11, pp. 1161-1167, 2011.

[6] A. Scalbert, C. Manach, C. Morand, C. Rémésy, and L. Jiménez, "Dietary polyphenols and the prevention of diseases," Critical Reviews in Food Science and Nutrition, vol. 45, no. 4, pp. 287-306, 2005.
[7] P. Van den Brandt, "The impact of a Mediterranean diet and healthy lifestyle on premature mortality in men and women," American Journal of Clinical Nutrition, vol. 94, pp. 913-920, 2011.

[8] A. Kondrashov, R. Ševčík, H. Benáková, M. Koštírová, and S. Štípek, "The key role of grape variety for antioxidant capacity of red wines," e-SPEN, The European e-Journal of Clinical Nutrition and Metabolism, vol. 4, no. 1, pp. e41-e46, 2009.

[9] A. P. Gollücke, "Recent applications of grape polyphenols in foods, beverages and supplements," Recent Patents on Food, Nutrition \& Agriculture, vol. 2, no. 2, pp. 105-109, 2010.

[10] M. Iriti and F. Faoro, "Bioactivity of grape chemicals for human health," Natural Product Communications, vol. 4, no. 5, pp. 611-634, 2009.

[11] J. F. Leikert, T. R. Räthel, P. Wohlfart, V. Cheynier, A. M. Vollmar, and V. M. Dirsch, "Red wine polyphenols enhance endothelial nitric oxide synthase expression and subsequent nitric oxide release from endothelial cells," Circulation, vol. 106, no. 13, pp. 1614-1617, 2002.

[12] T. R. Räthel, R. Samtleben, A. M. Vollmar, and V. M. Dirsch, "Activation of endothelial nitric oxide synthase by red wine polyphenols: impact of grape cultivars, growing area and the vinification process," Journal of Hypertension, vol. 25, no. 3, pp. 541-549, 2007.

[13] C. Auger, M. Chaabi, E. Anselm, A. Lobstein, and V. B. Schini-Kerth, "The red wine extract-induced activation of endothelial nitric oxide synthase is mediated by a great variety of polyphenolic compounds," Molecular Nutrition and Food Research, vol. 54, no. 2, supplement, pp. S171-S183, 2010.

[14] V. B. Schini-Kerth, N. Étienne-Selloum, T. Chataigneau, and C. Auger, "Vascular protection by natural product-derived polyphenols: in vitro and in vivo evidence," Planta Medica, vol. 77, no. 11, pp. 1161-1167, 2011.

[15] S. V. F. Madeira, C. Auger, E. Anselm et al., "eNOS activation induced by a polyphenol-rich grape skin extract in porcine coronary arteries," Journal of Vascular Research, vol. 46, no. 5, pp. 406-416, 2009.

[16] R. Masella, R. Di Benedetto, R. Varì, C. Filesi, and C. Giovannini, "Novel mechanisms of natural antioxidant compounds in biological systems: involvement of glutathione and glutathione-related enzymes," Journal of Nutritional Biochemistry, vol. 16, no. 10, pp. 577-586, 2005.

[17] M. S. Fernández-Pachón, G. Berná, E. Otaolaurruchi, A. M. Troncoso, F. Martín, and M. C. García-Parrilla, "Changes in antioxidant endogenous enzymes (activity and gene expression levels) after repeated red wine intake," Journal of Agricultural and Food Chemistry, vol. 57, no. 15, pp. 6578-6583, 2009.

[18] R. Rodrigo, A. Miranda, and L. Vergara, "Modulation of endogenous antioxidant system by wine polyphenols in human disease," Clinica Chimica Acta, vol. 412, no. 5-6, pp. 410-424, 2011.

[19] R. J. Nijveldt, E. Van Nood, D. E. C. Van Hoorn, P. G. Boelens, K. Van Norren, and P. A. M. Van Leeuwen, "Flavonoids: a review of probable mechanisms of action and potential applications," American Journal of Clinical Nutrition, vol. 74, no. 4, pp. 418-425, 2001.

[20] S. Frías, J. P. Pérez Trujillo, E. M. Peńa, and J. E. Conde, "Classification and differentiation of bottled sweet wines of Canary Islands (Spain) by their metallic content," European Food Research and Technology, vol. 213, no. 2, pp. 145-149, 2001.

[21] J. P. W. Houtman, "Trace elements and cardiovascular diseases," Journal of Cardiovascular Risk, vol. 3, no. 1, pp. 18-25, 1996. 
[22] B. Bao, A. S. Prasad, F. W. J. Beck et al., "Zinc decreases C-reactive protein, lipid peroxidation, and inflammatory cytokines in elderly subjects: a potential implication of zinc as an atheroprotective agent," American Journal of Clinical Nutrition, vol. 91, no. 6, pp. 1634-1641, 2010.

[23] S. Frassinetti, G. L. Bronzetti, L. Caltavuturo, M. Cini, and C. D. Croce, "The role of zinc in life: a review," Journal of Environmental Pathology, Toxicology and Oncology, vol. 25, no. 3, pp. 597-610, 2006.

[24] M. Stefanidou, C. Maravelias, A. Dona, and C. Spiliopoulou, "Zinc: a multipurpose trace element," Archives of Toxicology, vol. 80, no. 1, pp. 1-9, 2006.

[25] O. Pechánová, I. Bernátová, P. Babál et al., "Red wine polyphenols prevent cardiovascular alterations in L-NAME-induced hypertension," Journal of Hypertension, vol. 22, no. 8, pp. 1551-1559, 2004.

[26] J. Duarte, E. Andriambeloson, M. Diebolt, and R. Andriantsitohaina, "Wine polyphenols stimulate superoxide anion production to promote calcium signaling and endothelialdependent vasodilatation," Physiological Research, vol. 53, no. 6, pp. 595-602, 2004.

[27] I. Bernátová, O. Pechánová, P. Babál, S. Kyselá, S. Stvrtina, and R. Andriantsitohaina, "Wine polyphenols improve cardiovascular remodeling and vascular function in NO-deficient hypertension," American Journal of Physiology, vol. 282, no. 3, pp. H942-H948, 2002.

[28] V. L. Singleton, R. Orthofer, and R. M. Lamuela-Raventós, "Analysis of total phenols and other oxidation substrates and antioxidants by means of folin-ciocalteu reagent," Methods in Enzymology, vol. 299, pp. 152-178, 1998.

[29] D. S. Bredt and S. H. Snyder, "Isolation of nitric oxide synthetase, a calmodulin-requiring enzyme," Proceedings of the National Academy of Sciences of the United States of America, vol. 87, no. 2, pp. 682-685, 1990.

[30] O. Pecháňová, I. Bernátová, V. Pelouch, and F. Šimko, “Protein remodelling of the heart in NO-deficient hypertension: the effect of captopril," Journal of Molecular and Cellular Cardiology, vol. 29, no. 12, pp. 3365-3374, 1997.

[31] O. Pecháňová, J. Zicha, L. Paulis et al., "The effect of Nacetylcysteine and melatonin in adult spontaneously hypertensive rats with established hypertension," European Journal of Pharmacology, vol. 561, no. 1-3, pp. 129-136, 2007.

[32] R. Re, N. Pellegrini, A. Proteggente, A. Pannala, M. Yang, and C. Rice-Evans, "Antioxidant activity applying an improved ABTS radical cation decolorization assay," Free Radical Biology and Medicine, vol. 26, no. 9-10, pp. 1231-1237, 1999.

[33] T. J. Guzik and K. M. Channon, "Measurement of vascular reactive oxygen species production by chemiluminescence," in Hypertension Methods and Protocols, pp. 73-89, Humana Press, 2005.

[34] A. Lugasi and J. Hóvári, "Antioxidant properties of commercial alcoholic and nonalcoholic beverages," Nahrung, vol. 47, no. 2, pp. 79-86, 2003.

[35] J. Burns, P. T. Gardner, J. O’Neil et al., "Relationship among antioxidant activity, vasodilation capacity, and phenolic content of red wines," Journal of Agricultural and Food Chemistry, vol. 48, no. 2, pp. 220-230, 2000.

[36] J. H. Beattie and I. S. Kwun, "Is zinc deficiency a risk factor for atherosclerosis?" British Journal of Nutrition, vol. 91, no. 2, pp. 177-181, 2004.

[37] M. P. Cuajungco and K. Y. Fagét, "Zinc takes the center stage: its paradoxical role in Alzheimer's disease," Brain Research Reviews, vol. 41, no. 1, pp. 44-56, 2003.
[38] F. Kosar, I. Sahin, C. Taskapan et al., "Trace element status (Se, $\mathrm{Zn}, \mathrm{Cu}$ ) in heart failure," Anadolu Kardiyoloji Dergisi, vol. 6, pp. 216-220, 2006.

[39] J. P. W. Houtman, "Trace elements and cardiovascular diseases," Journal of Cardiovascular Risk, vol. 3, no. 1, pp. 18-25, 1996.

[40] B. Bao, A. S. Prasad, F. W. J. Beck et al., "Zinc decreases Creactive protein, lipid peroxidation, and inflammatory cytokines in elderly subjects: a potential implication of zinc as an atheroprotective agent," American Journal of Clinical Nutrition, vol. 91, no. 6, pp. 1634-1641, 2010.

[41] S. Frassinetti, G. L. Bronzetti, L. Caltavuturo, M. Cini, and C. D. Croce, "The role of zinc in life: a review," Journal of Environmental Pathology, Toxicology and Oncology, vol. 25, no. 3, pp. 597-610, 2006.

[42] M. Stefanidou, C. Maravelias, A. Dona, and C. Spiliopoulou, "Zinc: a multipurpose trace element," Archives of Toxicology, vol. 80, no. 1, pp. 1-9, 2006.

[43] P. Bhalla, R. Dhar, D. K. Dhawan, and V. Dam Chadha, "Neuroprotective effects of zinc on antioxidant defense system in lithium treated rat brain," Indian Journal of Experimental Biology, vol. 45, no. 11, pp. 954-958, 2007.

[44] T. Fukai and M. Ushio-Fukai, "Superoxide dismutases: role in redox signaling, vascular function, and diseases," Antioxidants and Redox Signaling, vol. 15, no. 6, pp. 1583-1606, 2011.

[45] The Jackson Laboratory, "Chemical Treatment Slows ALS in SOD1 Mouse," JAXNOTES Issue 516, 2010, http://jaxmice .jax.org/jaxnotes/516/516b.html.

[46] E. Mocchegiani, M. Muzzioli, and R. Giacconi, "Zinc and immunoresistance to infection in aging: new biological tools," Trends in Pharmacological Sciences, vol. 21, no. 6, pp. 205-208, 2000.

[47] M. H. Zou, C. Shi, and R. A. Cohen, "Oxidation of the zincthiolate complex and uncoupling of endothelial nitric oxide synthase by peroxynitrite," Journal of Clinical Investigation, vol. 109, no. 6, pp. 817-826, 2002.

[48] U. Förstermann, "Nitric oxide and oxidative stress in vascular disease," European Journal of Physiology, vol. 459, no. 6, pp. 923-939, 2010.

[49] R. López-Sepúlveda, R. Jiménez, M. Romero et al., "Wine polyphenols improve endothelial function in large vessels of female spontaneously hypertensive rats," Hypertension, vol. 51, no. 4, pp. 1088-1095, 2008.

[50] N. K. H. Khoo, C. R. White, L. Pozzo-Miller et al., "Dietary flavonoid quercetin stimulates vasorelaxation in aortic vessels," Free Radical Biology and Medicine, vol. 49, no. 3, pp. 339347, 2010.

[51] S. L. Chan, A. Tabellion, D. Bagrel, C. Perrin-Sarrado, C. Capdeville-Atkinson, and J. Atkinson, "Impact of chronic treatment with red wine polyphenols (RWP) on cerebral arterioles in the spontaneous hypertensive rat," Journal of Cardiovascular Pharmacology, vol. 51, no. 3, pp. 304-310, 2008.

[52] I. P. G. Botden, R. Draijer, B. E. Westerhof et al., "Red wine polyphenols do not lower peripheral or central blood pressure in high normal blood pressure and hypertension," American Journal of Hypertension, vol. 25, no. 6, pp. 718-723, 2012. 


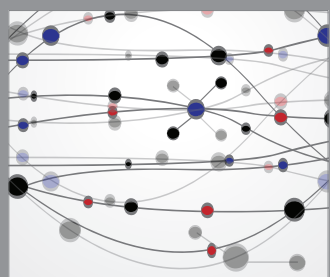

The Scientific World Journal
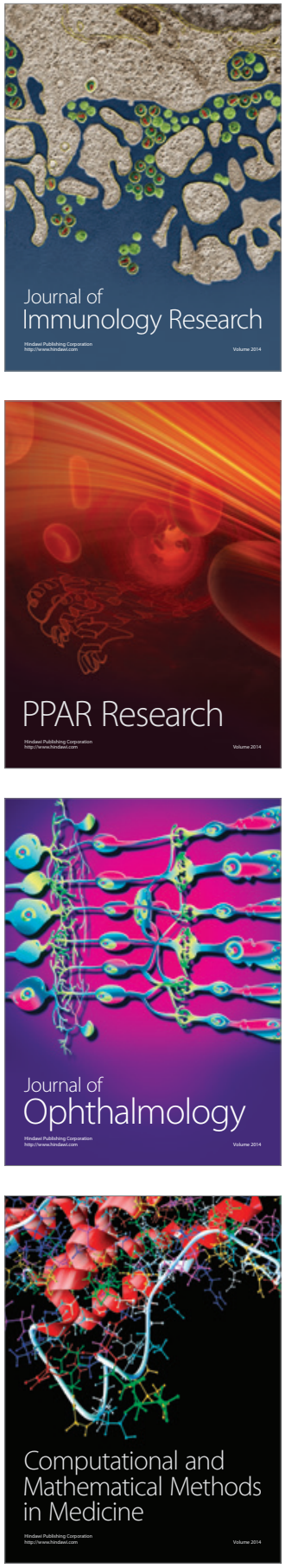

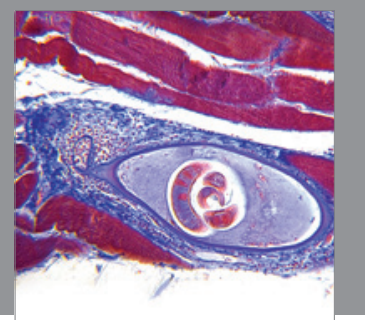

Gastroenterology

Research and Practice
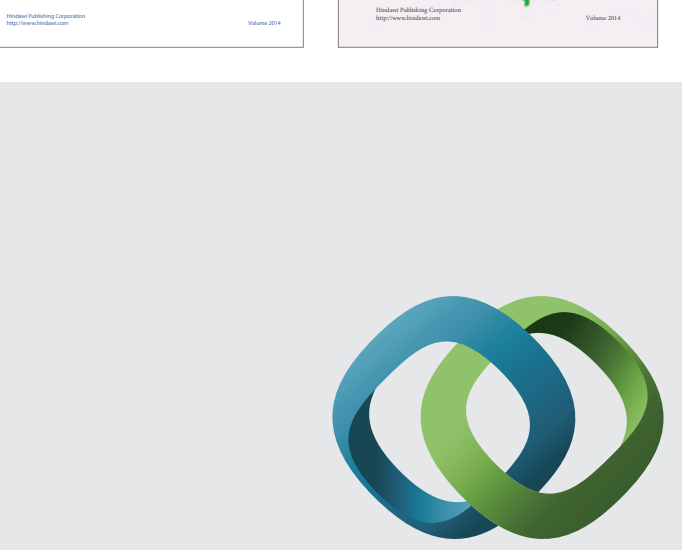

\section{Hindawi}

Submit your manuscripts at

http://www.hindawi.com
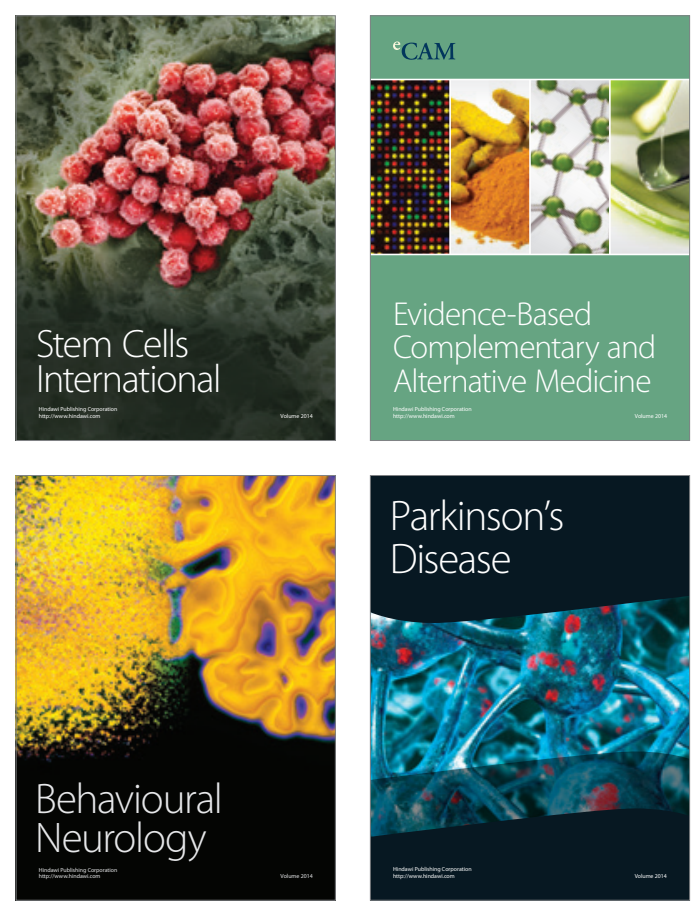

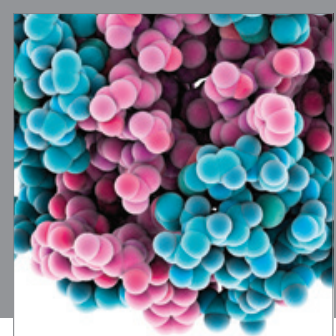

Journal of
Diabetes Research

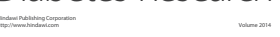

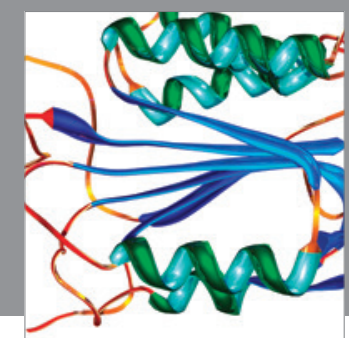

Disease Markers
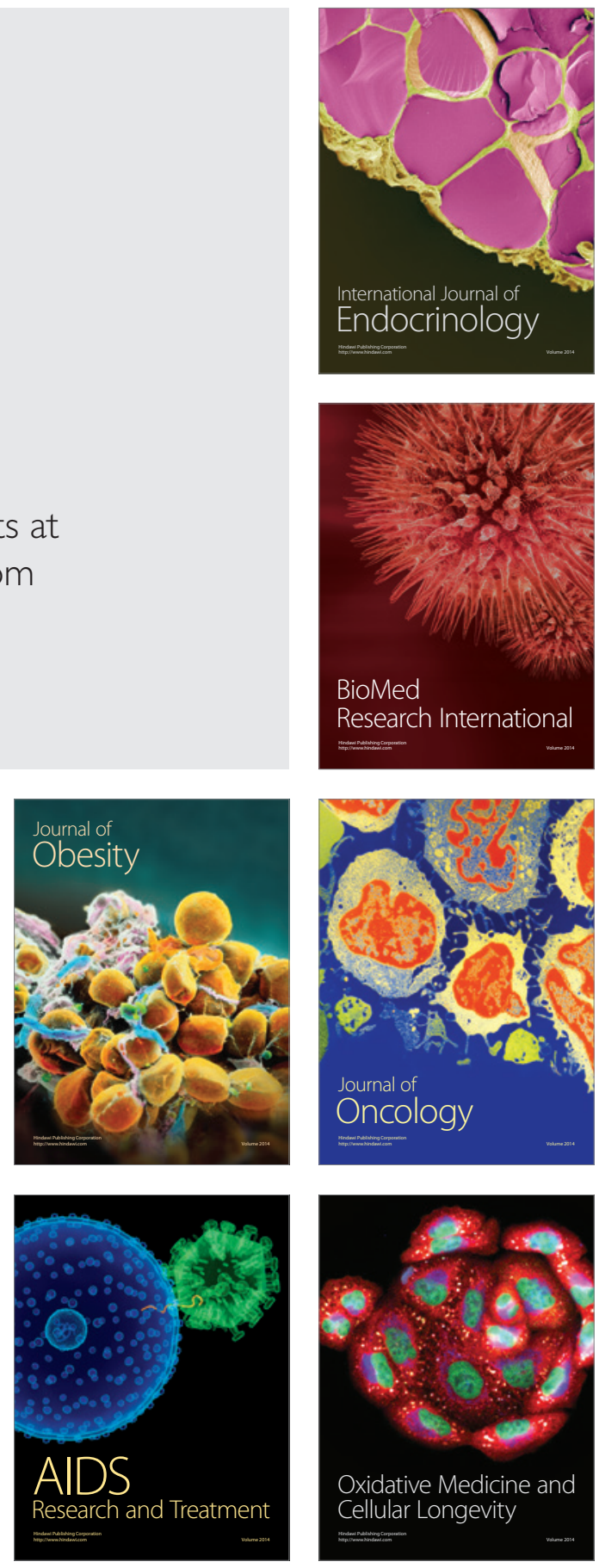